Texture of Crystalline Solids, 1980, Vol. 4, pp. 63-71 0390-7951/80/0402-0063\$04.50/0

(c) 1980 Gordon and Breach, Science Publishers, Inc.

Printed in the United States of America

\title{
CORRECTION OF INTEGRATED X-RAY INTENSITIES FOR PREFERRED ORIENTATION IN HEXAGONAL CLOSE-PACKED POWDERS USING EDXRD
}

\author{
J. KIVIL̈̈, E. LAINE and S. PARVIAINEN \\ Department of Physical Sciences, University of Turku, \\ 20500 Turku 50, Finland
}

(Received May 22, 1980)

\begin{abstract}
It is shown that energy-dispersive $\mathrm{x}$-ray diffraction (EDXRD) method can be used for correction of integrated intensities for preferred orientation in hexagonal close-packed powders. The theory is based upon examination of the polar axis density distribution and upon the use of hexagonal harmonics in its representation. The reflexion method by Schulz added with defocusing correction was used. Measurements were carried out on three zinc samples with different degrees of orientation, the largest correction being 54 percent.
\end{abstract}

\section{INTRODUCTION}

The theory of $x$-ray diffraction on powder samples assumes a completely random orientation of crystallites. In practice this demand is seldom realized. Therefore, the correction of integrated intensities for preferred orientation is necessary, when accurate intensity measurements are required.

Järvinen, Merisalo, Pesonen and Inkinen ${ }^{1}$ and Pesonen, Järvinen and Kurki-Suonio ${ }^{2}$ have developed for this purpose the symmetrized harmonic method and applied it successfully to cubic and hexagonal close-packed crystals. Later Laine, Kivilä and Lähteenmäki ${ }^{3}$ have applied this method to the study of preferred orientation in cubic powders using the energydispersive x-ray diffraction (EDXRD) method.

The purpose of this work is to test the EDXRD-method to studying of the preferred orientation in hexagonal closepacked powder samples and to determine the correction of integrated intensities. The theory is based upon examination of the polar axis density distribution and upon the use of hexagonal harmonics in its representation. 
2. THEORY

Järvinen et al. ${ }^{1}$ presented that the observed integrated intensity Iobsd, the polar axis density $W\left(\nu_{h k l}, \phi_{h k l}\right)$ and the intensity $I_{r a n}^{h k l}$ corrected for preferred orientation are related to each other by the expression

$$
I_{\operatorname{ran}}^{h k l}=I_{o b s d}^{h k I} / W\left(\nu_{h k l}, \phi_{h k l}\right) \text {, }
$$

where $v_{\text {hkl }}, \phi_{\text {hkl }}$ are the conventional spherical coordinates of the scattering vector with respect to the internal crystallographic axes. For an ideal sample the orientation distribution is completely random, corresponding to the polar axis density $W\left(\nu_{h k l}, \phi_{h k l}\right)=1$ and hence $I_{r a n}^{h k l}=I_{o b s d}^{h k l} \cdot$ Furthermore, the polar axis density can be given by

$$
W(\nu, \phi)=\sum_{i j} C_{i j} K_{i j}(\nu, \phi),
$$

where $K_{i j}$ are the symmetrized harmonics for the relevant point-group symmetry and $C_{i j}$ are the coefficients to be determined experimentally. By integration of the expression

(2) of the polar axis density, $W(\nu, \phi)$, we can write

$$
\operatorname{Ihkl}(\alpha)=\operatorname{Thkl} \sum_{i j} C_{i j} K_{i j}\left(\nu_{h k l}, \phi_{h k l}\right) P_{i}(\cos \alpha),
$$

where the parameter $T^{h k l}=I_{r a n}^{h k l}(0)$ and $P_{i}(\cos \alpha)$ is the Legendre polynomial of the order $i$. The values of the harmonic functions, $\mathrm{K}_{i j}$, and of the Legendre polynomials have been calculated. Thus by measuring the integrated intensities of the texture specimen $I^{h k l}(\alpha)$ for different reflexions as a function of polar angle, $\alpha$, the unknown coefficients, $C_{i j}$, and the parameter $T^{h k l}$, can be solved with Equation (3). By substituting these into Equation (2), we obtain the polar axis density. After that the correction of integrated $x$-ray intensities for preferred orientation can be made according to Equation (1).

In deriving Equation (3) no assumptions have been made about the crystal structure of the material studied. In the case of hexagonal close-packed crystals, the harmonic functions $K_{i j}$ are the hexagonal harmonics. Pesonen et al. ${ }^{2}$ concluded that when the orientation is not strong, it is sufficient to calculate the polar axis density to determine the coefficients $C_{i j}$ corresponding to $i \leq 10$. Thus we obtain nine different hexagonal harmonics ${ }^{2}$ 


$$
\begin{aligned}
& \mathrm{K}_{10}=\mathrm{P}_{i}(\cos v) ; i=0,2,4,6,8,10 \\
& \mathrm{~K}_{61}=\sin ^{2} v \cos 6 \phi \\
& \mathrm{K}_{81}=\frac{2000}{2401}\left(15 \cos ^{2} v-1\right) \mathrm{K}_{61} \\
& \mathrm{~K}_{10,1}=0.24242\left(323 \cos ^{4} v-102 \cos ^{2} v+3\right) \mathrm{K}_{61},
\end{aligned}
$$

where $\nu$ and $\phi$ are the spherical coordinates. In the case of the hexagonal crystals cos $\nu$ can be calculated from the equation

$\cos v=\frac{h_{1} h_{2}+k_{1} k_{2}+\frac{1}{2}\left(h_{1} k_{2}+h_{2} k_{1}\right)+\frac{3}{4} \frac{a^{2}}{c^{2}} l_{1} l_{2}}{\left[\left(h_{1}^{2}+k_{1}^{2}+h_{1} k_{1}+\frac{3}{4} \frac{a^{2}}{c^{2}} l_{1}^{2}\right)\left(h_{2}^{2}+k_{2}^{2}+h_{2} k_{2}+\frac{3}{4} \frac{a^{2}}{c^{2}} I_{2}^{2}\right)\right]^{\frac{1}{2}}}$.

In Table I, the values calculated of hexagonal harmonics for the first six reflexions of zinc are given.

The accuracy of the coefficients $C_{i j}$ obtained from the measurement of a certain reflexion hkl depends on the values of the functions $K_{i j}\left(\nu_{h k l}, \phi h_{k l}\right)$. I when $\left|K_{i j}\right|$ of the reflexion hkl is as close to unity as possible, $C_{i j}$ is then the most accurate. We can see from Table $I$, that by choosing the reflexions 100,002 and 110 , the coefficients $C_{i j}$ thus obtained were the most accurate.

\section{EXPERIMENTAL}

As a test material the zinc powder was used. The particle size was from 12-18 $\mu \mathrm{m}$. According to microscopic examination the shape of the particles was elongated. Three specimens were prepared by the compression of powder into a cylindrical sample chamber (diameter $13 \mathrm{~mm}$ ), applying pressures of $6 \mathrm{MPa}$ (sample 1), $210 \mathrm{MPa}$ (sample 2) and $300 \mathrm{MPa}$ (sample 3). For decreasing the porosity the pressure chamber was evacuated. In spite of using as low pressure as possible ( $6 \mathrm{MPa})$, we were unable to prepare a sample free from orientation effects.

The measurements were carried out by the method originally proposed by Schulz ${ }^{4}$ using the energy-dispersive $x$-ray diffraction. ${ }^{3}$ A flat-faced specimen was placed in the sample holder of Siemens texture goniometer and the specimen was in polychromatic radiation of an x-ray tube (copper target, $40 \mathrm{kV}$ and $18 \mathrm{~mA}$ ). The symmetrical horizontal and vertical divergence of the primary beam were both $0.6^{\circ}$. The width and the height of the receiving slit were both $1.0^{\circ}$. The sample rotated about its own normal and it was also inclined with respect to the vertical plane at stepping intervals of $\Delta \alpha=10^{\circ}$ up to $\alpha= \pm 60^{\circ}$. The integrated intensities were measured by $\mathrm{Si}(\mathrm{Li})$-semiconductor detector. For optimizing

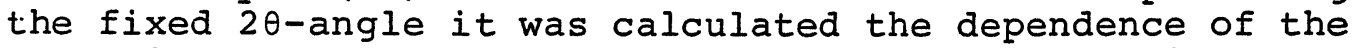
$2 \theta$-angle on photon energy for different hkl-reflexions of 


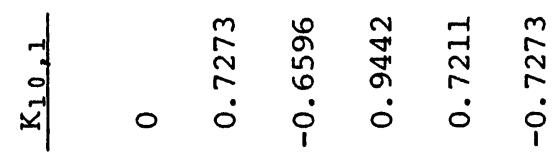

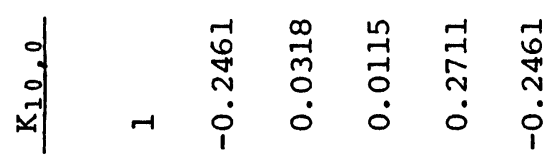

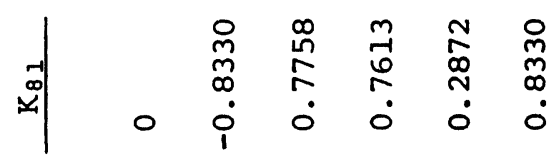

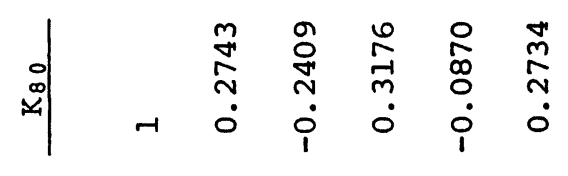

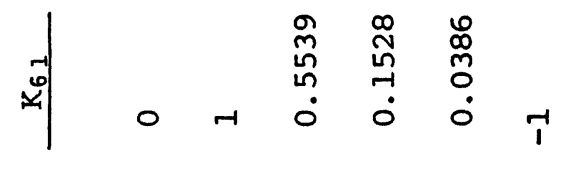

$$
\begin{aligned}
& \text { आI }
\end{aligned}
$$

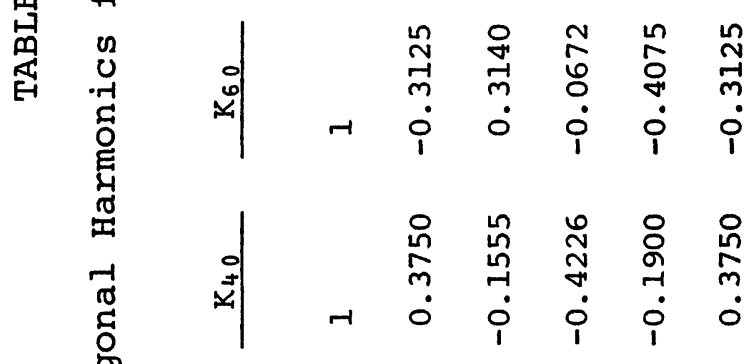

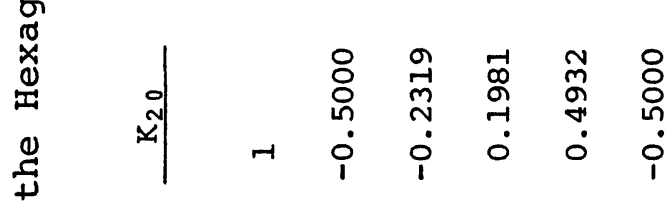

$$
\begin{aligned}
& \text { 出 의 }
\end{aligned}
$$

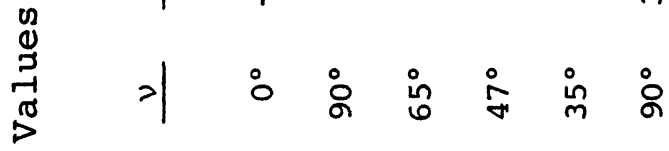

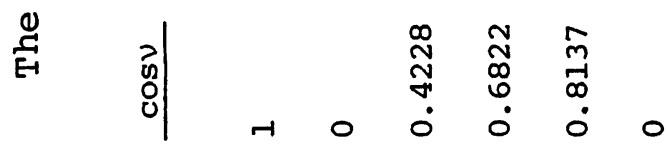

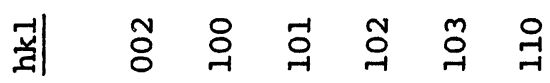


zinc. This dependence is shown in Figure 1 in which the energies of the fluorescence peaks can also be seen. On the

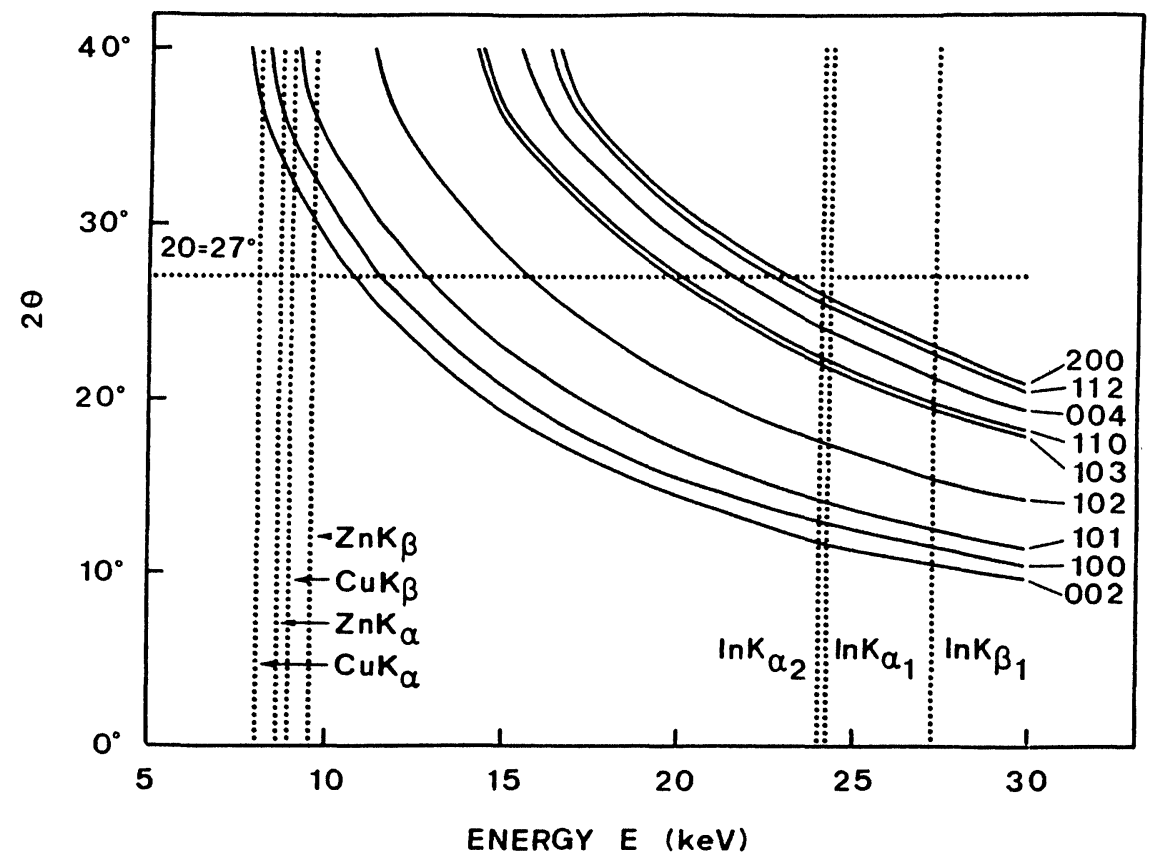

Figure 1. $2 \theta$ as a function of photon energy for different hkl values of zinc. $\operatorname{CuK}_{\alpha}, \operatorname{CuK}_{\beta}, \mathrm{ZnK}_{\alpha}, \mathrm{InK}_{\alpha 1,2}$ and $\mathrm{InK}_{\beta}$, are the harmful fluorescence peaks appearing in the energy-dispersive spectrum.

basis of Figure 1 the optimum value of $27^{\circ}$ for the fixed $2 \theta$-angle was obtained. The reflexions 002,100 and 101 were chosen to be examined. According to the theory the reflexion 110 would be slightly better in determining the coefficients $C_{i j}$ than the reflexion 101, but it overlaps badly with the reflexion 103.

With the Bragg angle mentioned above the reflexions 002 , 100 and 101 are diffracted at the energies $10.74 \mathrm{keV}, 11.50$ $\mathrm{keV}$ and $12.70 \mathrm{keV}$, respectively.

\section{RESULTS AND DISCUSSION}

The integrated intensities obtained were corrected by the symmetry and defocusing effects. We were unable to prepare a sample quite free from orientation effects and thus we could not determine the correction of defocusing experimentally. Therefore, the correction of defocusing was performed mathematically by means of equation derived by Gale and Griffiths ${ }^{5}$

$$
\frac{I(\alpha)}{I(0)}=\frac{\tau \sqrt{2}}{2 \operatorname{aerf} \frac{W}{2 \tau \sqrt{2}}}\left\{\alpha_{1} \operatorname{erf} \alpha_{1}-\alpha_{2} \operatorname{erf} \alpha_{2}+\frac{1}{\sqrt{\pi}}\left[\exp \left(-\alpha_{1}^{2}\right)-\exp \left(-\alpha_{2}^{2}\right)\right]\right\},
$$


where $\tau=\omega / \sqrt{2 \pi}$, $\omega$ is the integral breadth of the diffraction peak, when the polar angle $\alpha=0, \alpha_{1}=\left(a+\frac{1}{2} W\right) / \tau \sqrt{2}, \alpha_{2}=$ $\left(a-\frac{1}{2} W\right) / \tau \sqrt{2}$ and $a=(E / R) \cos \theta \tan \alpha$, where $w$ is the width of the receiving slit, $E$ is the height of the $x-r a y$ beam on the surface of the sample and $R$ is the distance of the primary slit from the sample. The integral breadths, $\omega$, were calculated from the diffraction spectra of sample 1. After being corrected for defocusing and symmetry, the intensity ratios $I^{h k l}(\alpha) / I^{h k l}(0)$, of the textured samples 2 and 3 are presented in Table II.

TABLE II

Corrected Intensity Ratios of Textured Samples 2 and 3 at Different Values of Polar Angle
$I^{002}(\alpha) / I^{002}$
(0)
$I^{100}(\alpha) / I^{100}$
(0)
$I^{101}(\alpha) / I^{1} 01$

\begin{tabular}{rccccccc}
$\frac{\alpha^{\circ}}{0}$ & Sample 2 & Sample 3 & Sample 2 & Sample 3 & Sample 2 & Sample 3 \\
\hline 0 & 1.000 & 1.000 & 1.000 & 1.000 & 1.000 & 1.000 \\
10 & 0.998 & 0.997 & 1.021 & 1.024 & 1.018 & 1.052 \\
20 & 0.995 & 0.997 & 1.072 & 1.047 & 1.059 & 1.102 \\
30 & 0.993 & 0.996 & 1.129 & 1.187 & 1.110 & 1.173 \\
40 & 0.899 & 0.881 & 1.299 & 1.252 & 1.168 & 1.257 \\
50 & 0.682 & 0.712 & 1.419 & 1.554 & 1.220 & 1.301 \\
60 & 0.602 & 0.544 & 1.593 & 1.705 & 1.282 & 1.393 \\
\hline
\end{tabular}

For the determination of the coefficients $c_{i j}$ and the parameters $\mathrm{T}^{\mathrm{hkl}}$, the measured and corrected (for defocusing and symmetry) integrated intensities were substituted into Equation (3). The Legendre polynomials $\mathrm{P}_{i}(\cos \alpha)$ and the hexagonal harmonics $K_{i j}$ are known. The coefficients $C_{i j}$ were determined by applying Equation (3) to the $I_{o b s d}^{\text {hkl }}(\alpha)$ values of the three reflexions 002,100 and 101. It has been shown ${ }^{2}$ that in the case of three favourable reflexions the $\alpha$ range could be reduced to $\alpha_{\max }=60^{\circ}$. The equation group was solved by the computer program.

The problem, how many terms we need to include from the series in Equation (3), was tested. The calculations were carried out by using the first three terms $(i \leq 4)$, the first four terms $(i \leq 6, j=0)$ and also the first nine terms $(i \leq 10, j=0,1)$ of the series. The inclusion of high order terms up to $i \leq 10$ increases the influence of statistical and systematic erros on the corrections. When only the first three terms were included, the corrections of preferred orientation were systematically too small. So, the limitation 
to the first four low order terms leads in this case to a more reliable correction of the preferred orientation. In Table III there are presented the coefficients $C_{i j}$ of the samples 2 and 3 determined in the way described above.

\section{TABLE III}

The Coefficients $C_{i j}$ of Samples 2 and 3 Determined from the Reflexions 002, 100 and 101 by Applying Equation (3) and Including the First Four Terms of the Series $(i \leq 6, j=0)$

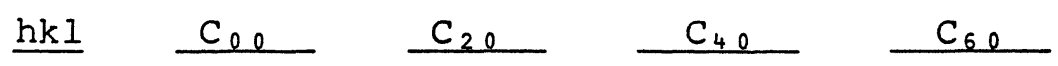

a) Sample 2

$\begin{array}{rrrrr}002 & 1.0000 & 0.7691 & -0.1664 & -0.0981 \\ 100 & 1.0000 & 0.7798 & 0.1012 & -0.0406 \\ 101 & 1.0000 & 0.8933 & -0.1280 & -0.0662\end{array}$

b) Sample 3

$\begin{array}{rrrrr}002 & 1.0000 & 0.8060 & -0.0722 & -0.1898 \\ 100 & 1.0000 & 0.9815 & 0.1404 & -0.0938 \\ 101 & 1.0000 & 1.0986 & -0.2253 & -0.1151\end{array}$

When the coefficients $C_{i j}$ have been solved, the polar axis density $W^{\text {hkl }}$ can be obtained from Equation (2). Thereafter the integrated intensities $\mathrm{I}^{\mathrm{hkl}}$ corrected for preferred orientation were calculated from Equation (1). Both the polar axis densities and the integrated intensities corrected for preferred orientation are presented in Table IV. In addition, in this table the observed intensities, when $\alpha=0$, and the parameter $\mathrm{T}^{\mathrm{hkl}}$ are included. The intensities have been normalized by choosing $I_{\text {obsd }}^{100}(0)=100$ for sample 3 . The correction for orientation $\Delta_{1}$ and the relative difference of the polar axis densities $\Delta_{2}=\left(I_{r a n}^{h k l}-T^{h k l}\right) / T^{h k l}$ are also given.

Looking at the polar axis densities it can be noticed that the compression-produced preferred orientation in hexagonal zinc powders is that in which the 002 plane tends to lie parallel to the surface of the specimen. Furthermore, in sample 3 prefereed orientation is a little stronger than in sample 2, as might be expected. Comparing the orientation in hexagonal zinc and in cubic aluminium measured previously, ${ }^{3}$ it is observed that the orientation effect is much stronger in 
TABLE IV

The Normalized Observed Intensities I obsd $(0)$, the Polar Axis Densities $\mathrm{W}^{\mathrm{hkl}}$, the Corrected Intensities $I_{r a n}^{h k l}$, the Parameter $T^{\text {hkl }}$, the

Corrections for Orientation $\Delta_{1}$ and the Relative

Differences of the Polar Axis Densities $\Delta_{2}$ for the

First Three Reflexions of Zinc Obtained

from Samples 2 and 3

\begin{tabular}{|c|c|c|c|c|c|c|}
\hline hkl & $I_{\text {obsd }}^{\text {hkI }}(0)$ & $\mathrm{w}^{\mathrm{hkl}}$ & $I_{\text {ran }}^{h k l}$ & $\mathrm{~T}^{\mathrm{hkl}}$ & $\Delta_{1} \frac{8}{8}$ & $\Delta_{2} 8$ \\
\hline a) & Sample 2 & & & & & \\
\hline 002 & 243.2 & 1.505 & 161.6 & 158.4 & -50.5 & 2.07 \\
\hline 100 & 105.5 & 0.661 & 159.7 & 161.0 & 33.9 & -0.87 \\
\hline 101 & 744.7 & 0.792 & 940.3 & 941.8 & 20.8 & -0.16 \\
\hline b) & Sample 3 & & & & & \\
\hline 002 & 257.0 & 1.544 & 166.4 & 163.2 & -54.4 & 2.01 \\
\hline 100 & 100.0 & 0.621 & 161.0 & 163.6 & 37.9 & -1.61 \\
\hline 101 & 705.0 & 0.744 & 947.6 & 970.6 & 25.6 & -2.37 \\
\hline
\end{tabular}

hexagonal zinc. Therefore, it is rather difficult in the case of hexagonal crystal symmetry to prepare a sample quite free from orientation effects. So it is very important to correct the measured integrated intensities from hexagonal powders for preferred orientation.

It can also be seen from Table IV that the relative differences of the polar axis densities are alz smaller than $2.5 \%$ in spite of the fact that the correction of the integrated intensities varied from $20.8 \%$ to $54.4 \%$.

The energy-dispersive $x$-ray diffraction (EDXRD) method shows to be well suited for the correction of the integrated $x-r a y$ intensities for preferred orientation in hexagonal powders. However, there are some difficulties such as the overlapping of the diffraction lines and the low intensity. Moreover, it is very difficult to prepare from hexagonal material a specimen free from orientation effects. In this case the defocusing correction must be performed using a mathematical calibration curve. Thus the correction of the integrated intensities of the hexagonal materials is more laborious than one of the cubic powders. ${ }^{3}$

If we compare the EDXRD method with the conventional angle-dispersive $x$-ray diffraction (ADXRD) method it is considerably more rapid, because several diffraction lines can 
be recorded simultaneously. The countrate and the statistics can still be increased using a synchrotron radiation as an $\mathrm{x}$-ray source. The adjustment of the texture goniometer system is easier in EDXRD than in ADXRD, because the Bragg angle is fixed and during one measurement the sample rotates only in its own plane ( $\psi$ rotation). It is not possible to omit the correction of defocusing by using a sufficiently large receiving slit, because then the diffraction lines often overlap.

The results obtained for powder samples of $z$ inc suggest that correction of the integrated intensities for preferred orientation using the EDXRD method is possible in the case of hexagonal crystal symmetry.

\section{REFERENCES}

1. M. Järvinen, M. Merisalo, A. Pesonen and O. Inkinen, J. Appl. Cryst., 3,313 (1970).

2. A. Pesonen, M. Järvinen and K. Kurki-Suonio, Phys. Fenn., 8, 81 (1973).

3. E. Laine, J. Kivilä and I. Lähteenmäki, Texture, 2, 243 (1977).

4. I. Schulz, J. Appl. Phys., 20, 1030 (1949).

5. B. Gale and D. Griffiths, Brit. J. Appl. Phys., 11, 96 (1960). 